\title{
Correction to: Pharmacology of Inhaled Anesthetics
}

Elizabeth Demers Lavelle and Swamy Kurra

Correction to: E. Farag et al. (eds.), Basic Sciences in Anesthesia, - https://doi.org/10.1007/978-3-319-62067-1_10

The book was inadvertently published with abbreviation "MAP mean arterial pressure" which was additionally present in the footnote of Table 10.2. It has been removed with this correction chapter.

The updated online version of this chapter can be found at

- https://doi.org/10.1007/978-3-319-62067-1_10 